\title{
Enhancement of Dielectric Properties with the Addition of Bromine and Dopamine Modified Barium Titanate Particles to Silicone Rubber
}

\author{
Liang Jiang \\ Technological University Dublin \\ David Kennedy \\ Technological University Dublin, david.kennedy@tudublin.ie \\ Stephen Jerrams \\ Technological University Dublin, stephen.jerrams@tudublin.ie
}

See next page for additional authors

Follow this and additional works at: https://arrow.tudublin.ie/aegart

Part of the Chemistry Commons

\section{Recommended Citation}

Jiag, L, Kennedy, D., Jerrams, S. \& Betts, A. (2017). Enchancement of dielectric properties with the addition of bromine and dopamine modified barium titanate particles to silicone rubber. MRS Communications, vol. 6, no. 4, pg. 437-441. doi:10.1557/mrc.2016.53

This Article is brought to you for free and open access by the Applied Electrochemistry Group at ARROW@TU Dublin. It has been accepted for inclusion in Articles by an authorized administrator of ARROW@TU Dublin. For more information, please contact arrow.admin@tudublin.ie, aisling.coyne@tudublin.ie, gerard.connolly@tudublin.ie.

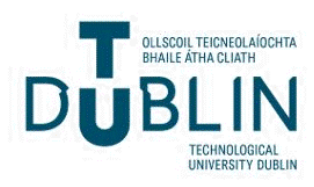




\section{Authors}

Liang Jiang, David Kennedy, Stephen Jerrams, and Tony Betts

This article is available at ARROW@TU Dublin: https://arrow.tudublin.ie/aegart/6 


\title{
Enhancement of dielectric properties with the addition of bromine and dopamine modified barium titanate particles to silicone rubber
}

\author{
Liang Jiang 1, a , David Kennedy ${ }^{\mathrm{c}}$, Stephen Jerrams ${ }^{\mathrm{a}}$, Anthony Betts ${ }^{\mathrm{b}}$ \\ ${ }^{a}$ Centre for Elastomer Research, Focas Research Institute, Dublin Institute of Technology, Dublin 8, \\ Ireland \\ ${ }^{\mathrm{b}}$ Applied Electrochemistry Group, Focas Research Institute, Dublin Institute of Technology, Dublin 8, \\ Ireland \\ ${ }^{c}$ Department of Mechanical Engineering, Dublin Institute of Technology, Dublin 1, Ireland
}

\begin{abstract}
Dual coated barium titanate (BT) particles were prepared using dopamine in conjunction with bromine in order to enhance the dielectric constant of silicone rubber (SR) composites containing evenly distributed BT particles. The results showed that both dopamine and bromine were deposited on the BT particles. The dielectric constant of the SR/BT composite was significantly increased from 3.6 to 4.7 at $1 \mathrm{kHz}$ with the addition of BT modified with dopamine (DP-BT). Moreover, the dielectric constant further rose to 4.9 at $1 \mathrm{kHz}$ when the DP-BT particle was grafted with bromine (Br-DP-BT). This facile process represents an efficient and convenient way of enhancing the dielectric properties of dielectric elastomer (DE) composites.
\end{abstract}

Keywords: Coating; Barium titanate; Composites; Dielectric elastomer

\section{Introduction}

Dielectric elastomers (DEs) can respond to electrical stimuli by changing their shape and belong to the family of smart materials [1-3]. Generally, DEs can induce several levels of strains (from 10 to $300 \%$ ), but they require large electric fields (usually around $100 \mathrm{~V} / \mu \mathrm{m}$ ) to achieve large actuated strains [4]. It has been reported that the operating electric field of DEs could be decreased for these materials by increasing the DEs' dielectric constants and also through lowering Young's modulus [5]. A soft silicone rubber (SR) based DE has the advantages of low Young's modulus combined with lower viscoelasticity and good thermal stability [6]. However, its deformability under an electric field is limited with a low inherent dielectric constant $(\approx 2.9)[2,6]$. The most popular approach to increasing the dielectric constant is by incorporating nano/micron sized high-dielectric filler particles into the DE matrix. Examples of fillers used are titanium dioxide $\left(\mathrm{TiO}_{2}\right)$ [7-9], lead magnesium niobate-lead titanate (PMN-PT) [10, 11], BT [12, 13] and functionalized graphene sheet (FGS) [14]. Ferroelectric oxides which exhibit a spontaneous electric polarization can also be reoriented in an external field. In particular, these oxides possess a large dielectric constant. Perovskite BT is one example of such oxides and exists as a white powder. It has a high dielectric constant of above 1200 [15]. Nevertheless, inorganic BT has poor compatibility with SR and this disadvantage prevents its further application in soft actuators. To negate this limitation, researchers focused on surface modification of BT $[13,16]$. Dopamine, which can interact strongly with metal oxides by forming hydrogen bonds [17], was considered an effective chemical with which to modify the surface of BT particles $[16,18]$. Moreover, the aromatic group, the hydrogen-bonded

${ }^{1}$ Corresponding author, email: liang.jiang@mydit.ie 
hydroxyl-dopamine species and the bromide group are of high polorisability [19-21] which is propitious to the enhancement of the dielectric constant. This research had the aim of enhancing the dielectric constant of SR based DE composites by adding the DP-BT and a further increase in the dielectric constant was sought by grafting bromine onto the DP-BT surface.

\section{Experimental}

\subsection{DP-BT and Br-DP-BT preparation}

Fig. 1 shows reactions for obtaining the DP-BT and the Br-DP-BT. The coating research on DPBT was reported previously [22]. In this work, $10 \mathrm{~g}$ of BT was dispersed in $50 \mathrm{ml}$ of ethnol-water mixture (with a mass ratio of 1:1) and stirred for $30 \mathrm{~min}$. Thus, $\mathrm{OH}^{-}$functional groups were added to the particle surfaces. The mixture was then poured into $450 \mathrm{ml}$ of deionized water with the addition of $1 \mathrm{~g}$ of dopamine hydrochloride. Thereafter, the suspension was stirred at $60{ }^{\circ} \mathrm{C}$ overnight prior to being subjected to ultrasonic shaking for $30 \mathrm{~min}$. The DP-BT coated particles were recovered by vacuum filtration. Subsequently they were washed using deionized water and finally dried at $60^{\circ} \mathrm{C}$ for one day in a vacuum to avoid oxidization of the dopamine.

For deposition of bromine on DP-BT particles, an approach of directly grafting the bromine on dopamine was employed [23]. The resulting DP-BT particles were initially ground up and dissolved in a mixture of $20 \mathrm{ml}$ acetic acid and $60 \mathrm{ml}$ chloroform. Subsequently $1.25 \mathrm{ml}$ concentrated bromine liquid was added and reacted overnight with magnetic stirring. The solution was heated at $60^{\circ} \mathrm{C}$ in order to remove the remnant bromine, acetic acid and chloroform. Finally, the powder was washed with deionized water and dried at $60^{\circ} \mathrm{C}$ for one day.

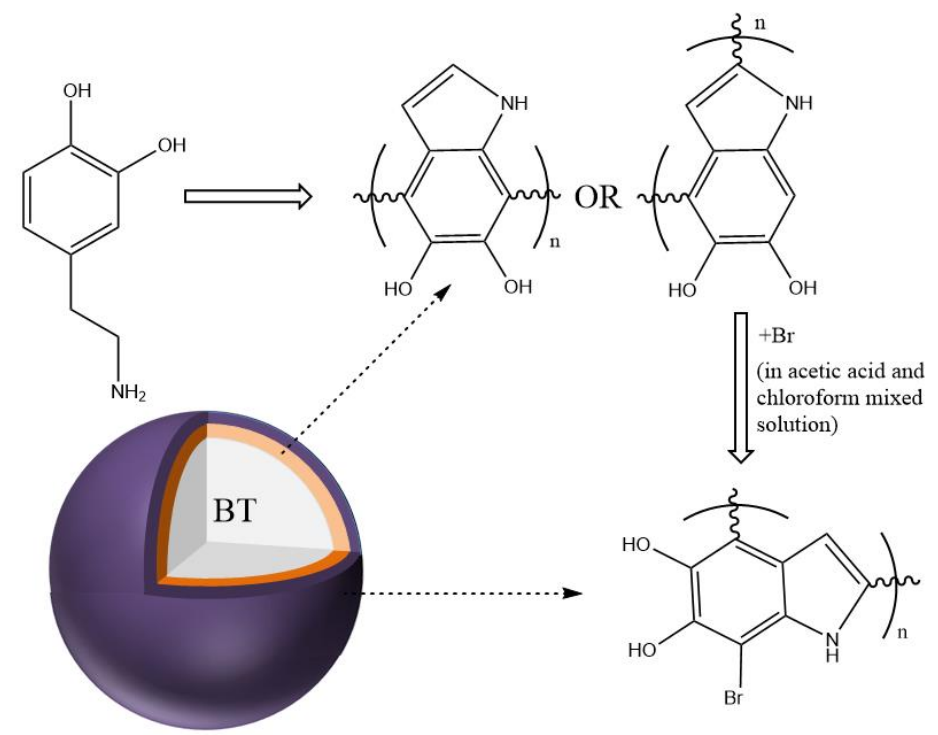

Fig. 1. Schematic diagrams of the fabrication of bromine and dopamine coated BT.

\subsection{DE films preparation}

Initially, the two part of SR were mixed at a weight ratio of 1:1 and dissolved in heptane. Then, the particles (BT, DP-BT and Br-DP-BT) with the required weight percentage of $10 \%$ were added to the mixture. In order to achieve uniform dispersion of the SR and fillers in heptane, the mixture 
was subjected to ultrasonic shaking for $20 \mathrm{~min}$. To complete the process, the mixture was poured into a watch glass, subjected to ultrasonic shaking for $30 \mathrm{~min}$ and heated for $3 \mathrm{~h}$ at $80{ }^{\circ} \mathrm{C}$ in a water bath with an ultrasonic cleaner which performed the function of heating and shaking under aerated conditions.

\subsection{Characterisation}

The morphological features of all particles in this work were observed using a Scanning Electron Microscope (SEM) (Zeiss Supra). Elemental analysis of these particles was carried out using an Energy Dispersive X-Ray Spectrometer (EDS) (Oxford Inca Xmax) which was coupled to the SEM. Fourier Transform Infrared Spectroscopy (FTIR) spectra of the micro-particles were recorded in the $350-4000 \mathrm{~cm}^{-1}$ range with a Perkin Elmer 400 Series Spectrometer. Dielectric measurements of DE films were conducted on a Turnkey broadband dielectric spectrometer at $20{ }^{\circ} \mathrm{C}$ in the frequency range of $10 \mathrm{~Hz}$ to $1 \mathrm{MHz}$.

\section{Results and Discussion}

Fig. 2 shows the SEM images of BT, DP-BT and Br-DP-BT particles. As shown in Fig. 2 (a) the BT particles were almost-spherical with a diameters of around $500 \mathrm{~nm}$. They formed clusters easily and the surfaces of these particles were relatively smooth. After coating with dopamine and bromine, the treated particles increased in diameter by more than $200 \mathrm{~nm}$ (refer to Fig. 2 (b) and Fig. 2 (c)). Additionally, the surfaces of the coated particles became rough as a result of being modified with dopamine and bromine reagents.

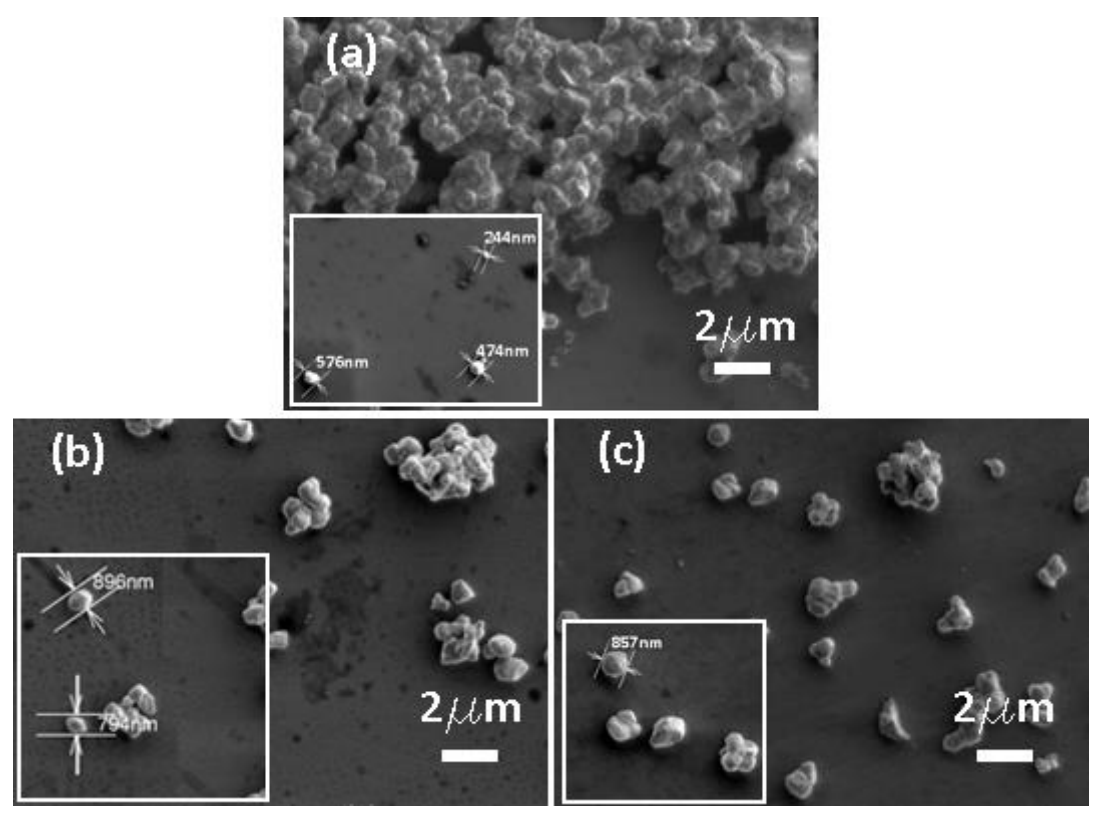

Fig. 2. SEM micrographs of particles used in the experiments: (a) BT; (b) DP-BT; (c) Br-DP-BT.

Fig. 3 shows the EDS spectrum of DP-BT and Br-DP-BT. Element Ba along with elements Ti and $\mathrm{O}$ can be observed in Fig. 3 (a) indicating the fundamental constituents of BT. Carbon (C) was also detected in each of the particles mainly due to residues present in various chamber surfaces such as vacuum pump and from sample surface migration and reaction with the electron beam 
forming a carbon rich environment [24]. Moreover, the contents of C in DP-BT (46 wt\%) and BrDP-BT (44 wt\%) are more than that in BT (35 wt \%) indicating the deposition of dopamine on BT particles (refer to Figs. 3 (b) and (c)). However, the content of element $\mathrm{O}$ changed only slightly after modification with dopamine and bromine with the $\mathrm{O}$ atomic mass fraction of dopamine nearly equal to that of BT (21 wt\%). Fig. 2 (c) shows the Br peak on the spectra indicating the deposition of $\mathrm{Br}$ on DP-BT particles.

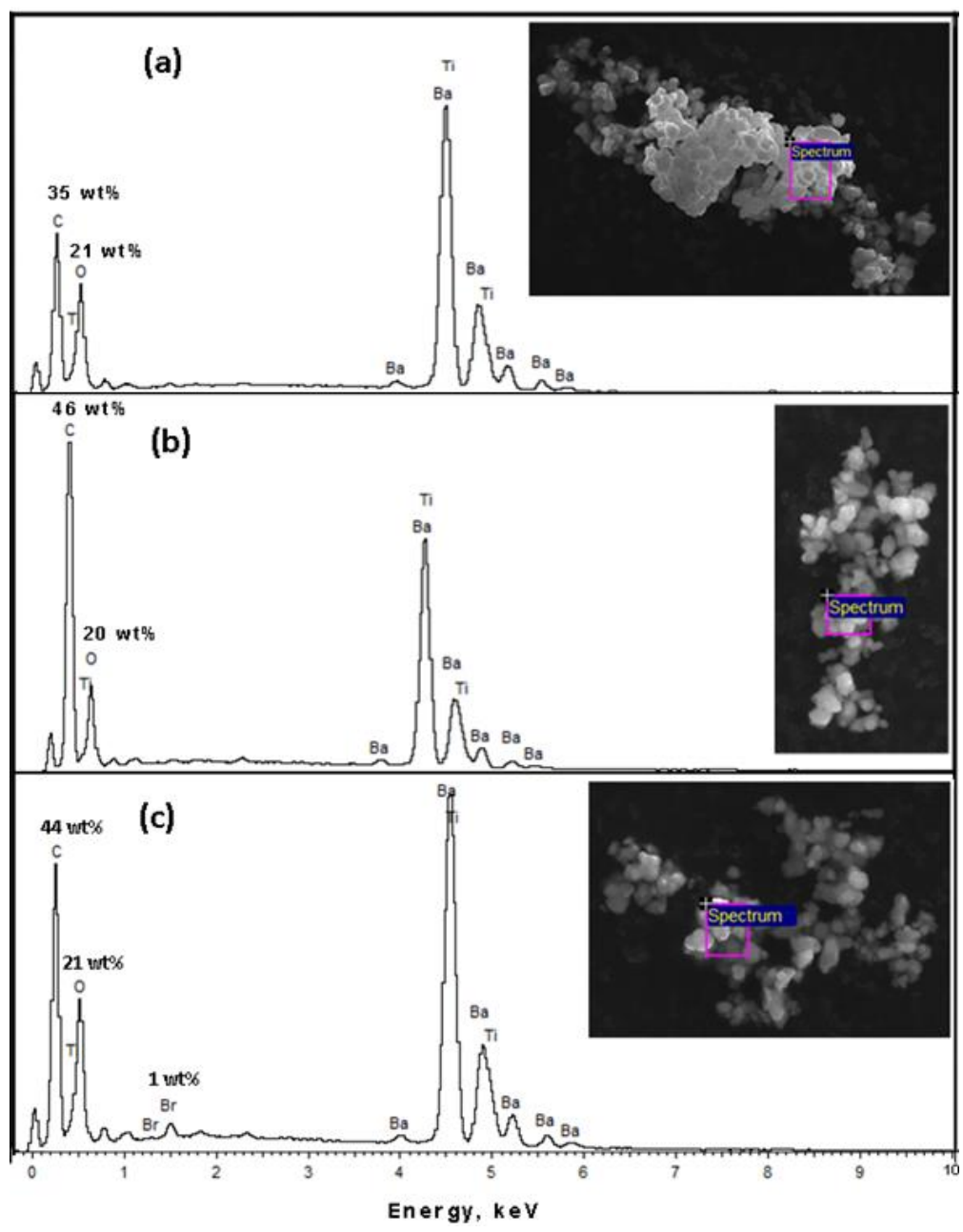

Fig. 3. EDS spectrum of DP-BT and Br-DP-BT.

Fig. 4 (a) shows the FTIR spectra of DP-BT and Br-DP-BT over a range of $500-600 \mathrm{~cm}^{-1}$. The peaks at 512 and 524 corresponding to the $\mathrm{C}-\mathrm{Br}$ stretch vibrations can be observed in the fingerprint region of the spectra. Fig.4 (b) shows the FTIR spectra of dopamine coated $\mathrm{BaTiO}_{3}$ (DP-BT) and bromine grafted DP-BT (Br-DP-BT) in the functional group region $(1000-4000$ $\left.\mathrm{cm}^{-1}\right)$. Due to the deposition of dopamine on $\mathrm{BaTiO}_{3}$, the $\mathrm{C}-\mathrm{H}$ bond appeared at $2916 \mathrm{~cm}^{-1}$. The transmittance peaks located at $1607 \mathrm{~cm}^{-1}$ and $1286 \mathrm{~cm}^{-1}$ corresponded to aromatic amine $\mathrm{N}-\mathrm{H}$ bending vibrations and $\mathrm{C}-\mathrm{N}$ stretching vibrations respectively. In addition, the appearance of the peaks at $2568 \mathrm{~cm}^{-1}$ and $2168 \mathrm{~cm}^{-1}$ was interpreted as indicating the presence of the $\mathrm{Ba}-\mathrm{OH}$ and Ti- 
$\mathrm{OH}$ formation [25] which was probably due to the addition of ethanol during the fabrication of DP-BT.
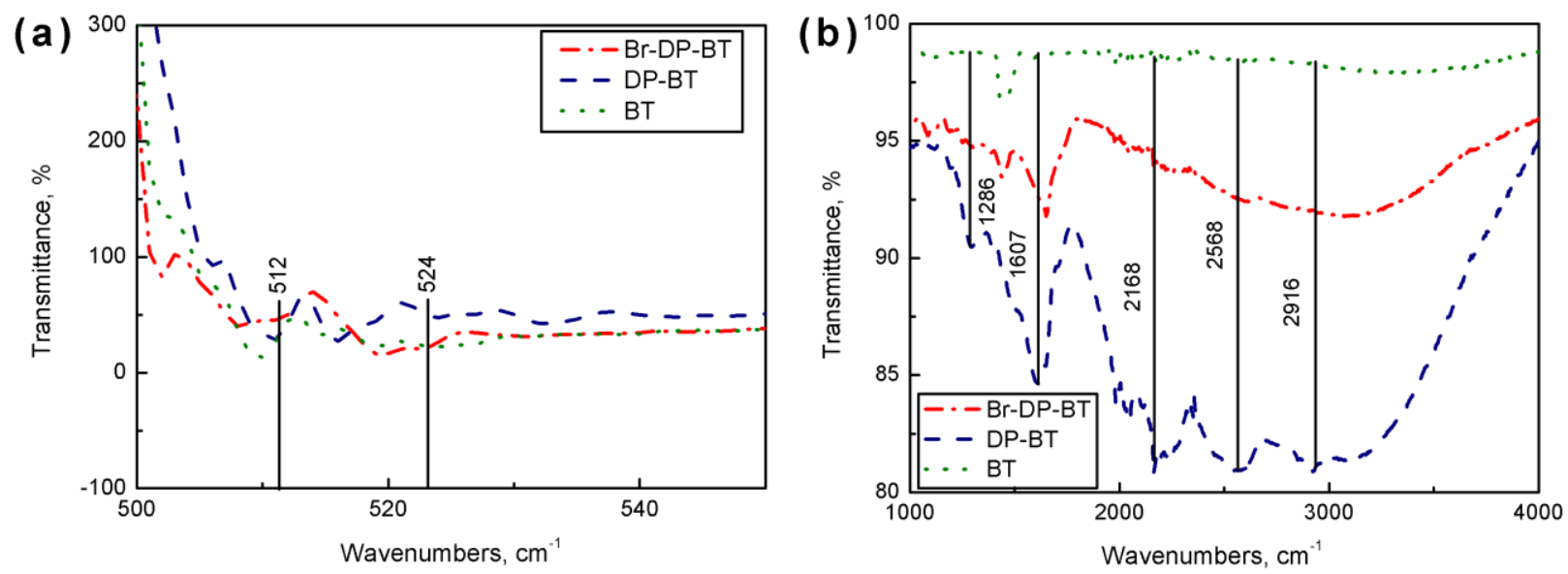

Fig.4. FTIR spectrum of DP-BT and Br-DP-BT in the frequency ranges of (a) $500-550 \mathrm{~cm}^{-1}$; (b)

$$
1000-4000 \mathrm{~cm}^{-1} \text {. }
$$

Fig. 5 shows the spectra of dielectric constant related to frequency for pure SR, SR/ 10wt\% BT composite, SR/10 wt\% DP-BT composite and SR/10 wt\% Br-DP-BT composite. As can be observed from Fig. 4, pure SR displayed a dielectric constant of about 2.9 at $1 \mathrm{kHz}$. The dielectric constant increased over the entire frequency range with the addition of $10 \mathrm{wt} \% \mathrm{BT}$ and to 3.4 at 1 $\mathrm{kHz}$. After adding the DP-BT particles in the SR matrix, the dielectric constant rose at all testing frequencies, most significantly to 4.7 at $1 \mathrm{kHz}$. Moreover, the dielectric constant was further enhanced by about 0.2 when SR was filled with $10 \mathrm{wt} \% \mathrm{Br}-\mathrm{DP}-\mathrm{BT}$ compared with that of SR/ 10 wt\% DP-BT composite. However, the dielectric constant changed only slightly in the wide test frequency range for each SR composite, probably because of the amorphous structure presented in SR which prevents the dielectric constant being influenced from the dipole orientaion in the electric field [26]. 


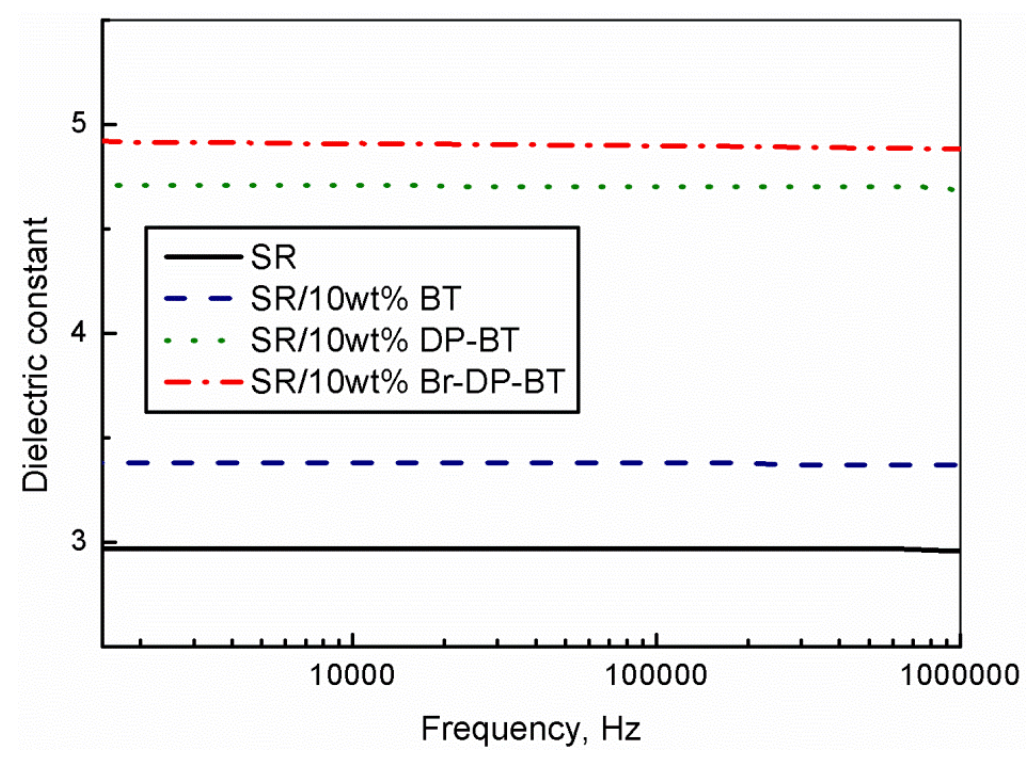

Fig. 5. Dependence of the dielectric constant on frequency.

\section{Conclusions}

This work illustrated an efficacious and relatively simple coating method for increasing the dielectric constants of SR/BT composites. SEM images showed the increases in diameter of the particles after they were coated with dopamine and bromine. It was clearly shown from the EDS and the FTIR spectrum results that dopamine and bromine were deposited on BT particles. Moreover, a dielectric constant of 4.9 was obtained by SR with $10 \mathrm{wt} \% \mathrm{Br}$-DP-BT which is numerically 2.5 higher than that of the SR/10 wt\% BT composite. Though, the dielectric constant of SR/10 wt\% Br-DP-BT composite rose slightly (0.2) by comparison with the SR/10 wt\% DP$\mathrm{BT}$, it was resumed that the dielectric constant should increase more significantly for higher filler loadings.

\section{Acknowledgements}

The authors gratefully acknowledge the support provided by the DIT Fiosraigh Dean of Graduate Students Award.

\section{References}

[1] R. Pelrine, R. Kornbluh, Q. Pei, J. Joseph, High-Speed Electrically Actuated Elastomers with Strain Greater Than 100\%, Science, 287 (2000) 836-839.

[2] P. Brochu, Q. Pei, Advances in Dielectric Elastomers for Actuators and Artificial Muscles, Macromolecular Rapid Communications, 31 (2010) 10-36.

[3] M. Giousouf, G. Kovacs, Dielectric elastomer actuators used for pneumatic valve technology, Smart Materials and Structures, 22 (2013) 6.

[4] L.J. Romasanta, M.A. Lopez-Manchado, R. Verdejo, Increasing the performance of dielectric elastomer actuators: A review from the materials perspective, Progress in Polymer Science, 51 (2015) 188-211. 
[5] R. Pelrine, R. Kornbluh, G. Kofod, High-Strain Actuator Materials Based on Dielectric Elastomers, Advanced Materials, 12 (2000) 1223-1225.

[6] R. Shankar, T.K. Ghosh, R.J. Spontak, Dielectric elastomers as next-generation polymeric actuators, Soft Matter, 3 (2007) 1116-1129.

[7] H.L. Liu, L.Q. Zhang, D. Yang, Y.C. Yu, L. Yao, M. Tian, Mechanical, Dielectric, and Actuated Strain of Silicone Elastomer Filled with Various Types of TiO2, Soft Mater., 11 (2013) 363-370.

[8] G. Ouyang, K. Wang, X.Y. Chen, TiO 2 nanoparticles modified polydimethylsiloxane with fast response time and increased dielectric constant, Journal of Micromechanics and Microengineering, 22 (2012) 074002.

[9] A.I. Kontos, A.G. Kontos, D.S. Tsoukleris, M.-C. Bernard, N. Spyrellis, P. Falaras, Nanostructured $\mathrm{TiO} 2$ films for DSSCS prepared by combining doctor-blade and sol-gel techniques, Journal of Materials Processing Technology, 196 (2008) 243-248.

[10] G. Gallone, F. Carpi, D. De Rossi, G. Levita, A. Marchetti, Dielectric constant enhancement in a silicone elastomer filled with lead magnesium niobate-lead titanate, Materials Science and Engineering: C, 27 (2007) 110-116.

[11] D. Yang, L. Zhang, H. Liu, Y. Dong, Y. Yu, M. Tian, Lead magnesium niobate-filled silicone dielectric elastomer with large actuated strain, Journal of Applied Polymer Science, 125 (2012) 2196-2201.

[12] D. Yang, F. Ge, M. Tian, N. Ning, L. Zhang, C. Zhao, K. Ito, T. Nishi, H. Wang, Y. Luan, Dielectric elastomer actuator with excellent electromechanical performance using slide-ring materials/barium titanate composites, Journal of Materials Chemistry A, 3 (2015) 9468-9479.

[13] P. Kim, S.C. Jones, P.J. Hotchkiss, J.N. Haddock, B. Kippelen, S.R. Marder, J.W. Perry, Phosphonic Acid-Modified Barium Titanate Polymer Nanocomposites with High Permittivity and Dielectric Strength, Advanced Materials, 19 (2007) 1001-1005.

[14] L. Romasanta, M. Hernández, M. López-Manchado, R. Verdejo, Functionalised graphene sheets as effective high dielectric constant fillers, Nanoscale Res Lett, 6 (2011) 1-6.

[15] W.S. Yun, J.J. Urban, Q. Gu, H. Park, Ferroelectric Properties of Individual Barium Titanate Nanowires Investigated by Scanned Probe Microscopy, Nano Letters, 2 (2002) 447-450.

[16] B.C. Luo, X.H. Wang, Y.P. Wang, L.T. Li, Fabrication, characterization, properties and theoretical analysis of ceramic/PVDF composite flexible films with high dielectric constant and low dielectric loss, J. Mater. Chem. A, 2 (2014) 510-519.

[17] M.-F. Lin, V.K. Thakur, E.J. Tan, P.S. Lee, Surface functionalization of BaTiO3 nanoparticles and improved electrical properties of $\mathrm{BaTiO} 3 /$ polyvinylidene fluoride composite, RSC Advances, 1 (2011) 576-578.

[18] D. Yang, M. Tian, D. Li, W. Wang, F. Ge, L. Zhang, Enhanced dielectric properties and actuated strain of elastomer composites with dopamine-induced surface functionalization, J. Mater. Chem. A, 1 (2013) 12276-12284.

[19] P. Ren, C. Wu, J.W. Ponder, Polarizable atomic multipole-based molecular mechanics for organic molecules, Journal of chemical theory and computation, 7 (2011) 3143-3161.

[20] R. Janoschek, E.G. Weidemann, H. Pfeiffer, G. Zundel, Extremely high polarizability of hydrogen bonds, Journal of the American Chemical Society, 94 (1972) 2387-2396.

[21] S. Greff, M. Zubia, G. Genta-Jouve, L. Massi, T. Perez, O.P. Thomas, Mahorones, Highly Brominated Cyclopentenones from the Red Alga Asparagopsis taxiformis, Journal of Natural Products, 77 (2014) 1150-1155. 
[22] L. Jiang, A. Betts, D. Kennedy, S. Jerrams, Improving the electromechanical performance of dielectric elastomers using silicone rubber and dopamine coated barium titanate, Materials \& Design, 85 (2015) 733-742.

[23] V.P. Shevchenko, I.Y. Nagaev, K.V. Shevchenko, M.G. Chernysheva, G.A. Badun, V.M. Fedoseev, N.F. Myasoedov, Solid-phase synthesis of deuterium- and tritium-labeled dopamine using carbon nanomaterials, Radiochemistry, 53 (2011) 336-340.

[24] P. Rolland, V. Carlino, R. Vane, Improved Carbon Analysis with Evactron Plasma Cleaning, Microscopy and Microanalysis, 10 (2004) 964-965.

[25] C. Racles, M. Cazacu, B. Fischer, D.M. Opris, Synthesis and characterization of silicones containing cyanopropyl groups and their use in dielectric elastomer actuators, Smart Materials and Structures, 22 (2013) 10.

[26] M. Sharma, G. Madras, S. Bose, Process induced electroactive [small beta]-polymorph in PVDF: effect on dielectric and ferroelectric properties, Physical Chemistry Chemical Physics, 16 (2014) 14792-14799. 